\title{
Impactos da Política na Acumulação da Riqueza dos Estados Brasileiros: a Contribuição dos Investimentos em Infraestrutura e dos Esforços em Inovação
}

\section{Impacts of Policy on the Wealth Accumulation by the Brazilian States: the Contribution of Infrastructure Investments and Innovation Efforts}

\author{
Leonardo Andrade Rocha* \\ Ahmad Saeed Khan** \\ Patricia V.P. Sales Lima* ** \\ Maria Ester Soares Dal Poz**** \\ Fernando Porfírio Soares de Oliveira* $* * *$
}

Resumo: O artigo busca analisar o impacto dos investimentos em infraestrutura e dos esforços em inovação na acumulação da riqueza dos estados. Foi adotado o método de regressão quantílica com o intuito de analisar o impacto dos investimentos em diferentes níveis de renda. Os resultados do modelo mostram que nas economias menos desenvolvidas e de baixa renda a ausência da infraestrutura torna o efeito do investimento superior em relação às economias mais desenvolvidas e com alta renda. Na medida em que a economia se aproxima da fronteira (a alta renda), o dinamismo dos negócios vai reduzindo os custos de oportunidade nos investimentos em Ciência $\mathcal{E}$ Tecnologia (CETT), alterando a demanda por mão de obra qualificada necessária para sustentar o deslocamento da fronteira. A ausência desta compreensão pode conduzir a uma alocação ineficiente dos recursos na geração de crescimento nos estados brasileiros.

Palavras-chave: Crescimento. Infraestrutura. Inovação. Desenvolvimento.

Abstract: The aim of this study is to analyze the impact of infrastructure investments and efforts in innovation in the accumulation of wealth of States. To test the assumptions, was adopted the quantile regression method in order to analyze the impact of investments in different income levels. The results of the model show that in less developed economies and

* Doutor em Economia pelo Instituto de Economia da Universidade Estadual de Campinas (Unicamp). Professor da Universidade Federal Rural do Semiárido (Ufersa). E-mail: leonardoandrocha@yahoo.com.br

** Ph.D. em Economia Agrícola e Recursos Naturais pela Oregon State University. Professor do Departamento de Economia Agrícola da Universidade Federal do Ceará (UFC). E-mail: saeed@ ufc.br

*** Doutora em Economia Aplicada pela Universidade de São Paulo - Escola Superior de Agricultura "Luiz de Queiroz" (ESALQ). Professora do Departamento de Economia Agrícola da UFC. E-mail: pvpslima@gmail.com

**** Doutora em Política Científica e Tecnológica pela Universidade Estadual de Campinas (Unicamp). Professora da Faculdade de Ciências Aplicadas da Unicamp. E-mail: ster.dalpoz@fca.unicamp.br

$* * * *$ Doutor em Administração pela Universidade Federal do Rio Grande do Norte. Professor da Ufersa. E-mail: fernandoporfiri@uol.com.br 
low income, lack of infrastructure makes the effect of the higher investment compared to more developed economies and with high income. According as the economy approaches the 'frontier' (high income), the business dynamic will reducing the opportunity costs in the investments in SET, changing the demand for skilled labor needed to sustain the displacement of the frontier. The absence of this understanding can lead to an inefficient allocation of resources in generating growth in Brazilian States.

Keywords: Growth. Infrastructure. Innovation. Development.

JEL Classification: $\mathrm{O} 1 ; \mathrm{O} 2 ; \mathrm{O} 4$.

\section{1 lntrodução}

Nos países em desenvolvimento, recentemente vêm-se apresentando um direcionamento da política na provisão de muitos bens e serviços tradicionalmente vistos como "bens públicos", com o intuito de sustentar o desenvolvimento, especialmente em momentos de instabilidade do cenário econômico. Esse direcionamento vinculado às recentes necessidades de avanço na tecnologia, em diversos setores, vem pautando um importante conflito na gestão dos recursos, notadamente em tais economias quando a restrição orçamentária do governo consiste em um claro gargalo para o desenvolvimento (ANG; MADSEN; 2011; CHATTERJEE; MORSHED, 2011.

Pesquisas recentes estimam que, entre 1990 e 2002, os investimentos governamentais em infraestrutura nos países em desenvolvimento representavam aproximadamente $70 \%$ do total da despesa empregada em infraestrutura, seguidos do setor privado com $25 \%$ e dos programas de assistência ao desenvolvimento com $5 \%$ (ESTACHE, 2004). A partir da década de 2000, os programas de reajuste fiscal juntamente com as promessas do setor privado restringiram os investimentos públicos em infraestrutura nos anos seguintes, comprometendo uma importante manutenção do crescimento (EASTERLY; SERVEN, 2003).

Segundo Easterly e Serven (2003),os atuais níveis de investimento em infraestrutura nas economias em desenvolvimento representam uma média de 40-50\% do que eram tais investimentos em 10 ou 15 anos atrás. No Brasil, a retração destes recursos equivalem a $174 \%$ do reajuste fiscal ao longo da última década, um cenário também visível nos países de renda baixa e média-baixa, demonstrando que os níveis de gastos são bem abaixo das necessidades estimadas.

Frente à realidade apresentada, nas economias em desenvolvimento encontra-se o Brasil, destacado com uma ampla assimetria regional ao longo do seu processo de desenvolvimento. Tal assimetria é responsável por diferentes resultados nos investimentos, de forma que recentes pesquisas vêm retratando este cenário para os estados brasileiros (ROCHA; SILVEIRA, 2009; ROCHA; KHAN; LIMA, 2013). 
Nesse sentido, Rocha e Silveira (2009) analisaram o impacto dos investimentos em infraestrutura e ciência $\mathcal{E}$ tecnologia (CETT) no crescimento do PIB dos estados brasileiros ao longo de 2002 a 2006. A hipótese levantada pelos autores sugere que, nos estados mais próximos da fronteira tecnológica, os investimentos em infraestrutura apresentam uma importância relativamente menor em relação aos investimentos em CETT, fato que ocorre em sentido inverso nos estados mais afastados. Para medir proximidade com a fronteira, os autores construíram um índice de produtividade do setor industrial e relativizaram a produtividade de cada estado com relação ao de maior produtividade. A interação do indicador de proximidade com cada investimento atribuiu pesos que, segundo o estudo, visa reduzir as distorções presentes entre as regiões. Como resultado, nos estados com maior proximidade, os investimentos em infraestrutura vão apresentando uma redução que vai sendo compensada com os investimentos em CETT.

Embora tais resultados sejam coerentes com outras aplicações, destacando-se Acemoglu, Aghion e Zilibotti (2006), Wu (2010) e Ang, Madsen e Islam (2011), a construção do indicador de proximidade com a fronteira presume que a importância do setor industrial seja a mesma para todos os estados. Ao contrário disto, diferentes composições são percebidas entre as regiões, transferindo importantes competências entre os setores. Este padrão pode comprometer parcialmente os resultados do modelo, à medida que analisamos as economias com intensa e baixa atividade industrial.

Visando comprovar tais hipóteses, a presente investigação busca analisar a contribuição dos investimentos em infraestrutura e dos esforços em inovação na acumulação da riqueza dos estados. Como uma alternativa à abordagem proposta por Rocha e Silveira (2009), esta pesquisa emprega o método de regressão quantílica com o intuito de analisar o impacto dos investimentos em diferentes níveis de renda. Assim, para as economias mais afastadas da fronteira, caracterizadas por um baixo nível de PIB per capita, a elasticidade parcial dos investimentos em infraestrutura é significativamente maior em relação aos gastos em CËT, vice-versa para as economias de alta renda (nos quantis superiores). Essa dualidade entre os investimentos evidencia que, nas diferentes regiões, a assimetria observada entre os níveis de renda condiciona a eficácia da política econômica.

A presente pesquisa está dividida em quatro partes, além desta introdução: na apresentação da abordagem teórica, iremos adaptar os modelos de Barro (1990), Alesina e Rodrik (1994) e Ortiz-Moctezuma et al. (2010). Considerando uma aplicação na teoria do controle ótimo, os gastos públicos em infraestrutura afetam a produtividade das firmas, de maneira similar a um insumo na produção. Esta relação condiciona a acumulação do capital no tempo, aumentando a produtividade das firmas em direção ao estadoestacionário. Entretanto, um efeito reverso do crescimento implica uma nova demanda por infraestrutura. Ao contrário 
de Ortiz-Moctezuma et al. (2010), somente uma parcela do crescimento implica novas demandas, de maneira que, nas soluções de estadoestacionário, o estoque de capital de longo prazo é afetado positivamente pelos gastos públicos e negativamente pela depreciação da infraestrutura.

Nas economias menos desenvolvidas e de baixa renda, a ausência da infraestrutura torna o efeito do investimento superior em relação às economias mais desenvolvidas e com alta renda. Neste caso, os investimentos em infraestrutura limitam-se à manutenção, alterando importantes custos de oportunidade com outros investimentos, como Pesquisa e Desenvolvimento.Na medida em que a economia se aproxima da fronteira, a alta renda, o dinamismo dos negócios vai reduzindo os custos de oportunidade nos investimentos em CETT, alterando a demanda por mão de obra qualificada necessária para sustentar o deslocamento da fronteira.

Na segunda parte, tem-se o método empírico, com a apresentação do modelo de regressão quantílica, considerando cada política estadual na contribuição do aumento da riqueza. A importância desse método consiste na caputura do efeito da heterogeneidade sobre a relação entre as variáveis, na medida em que se diferenciam os quantis da amostra. Recentes estudos, destacando-se Coad (2011) e Coad e Rao (2011), vêm apresentando o método de regressão quantílica como um importante instrumento de investigação destinado a analisar a relação entre variáveis econômicas quando incorporamos diferentes recortes na distribuição amostral.

A terceira parte consiste na apresentação dos resultados mostrando o efeito dual da política na proporção em que incorporamos as disparidades de renda como mecanismo de influência na relação das variáveis. Nas economias com baixa renda presentes nos quantis condicionais inferiores, a elasticidade dos investimentos em infraestrutura é maior em relação às economias de alta renda. Este padrão é observado de forma contrária em relação aos investimentos em CËT e no aumento da mão de obra com alta qualificação, medido pelo número de concluintes no ensino superior. Ao longo dos resultados, importantes estudos e a discussão com recentes pesquisas são confrontados de forma que validam as hipóteses elucidadas. Por último, têm-se as considerações finais do estudo com a principais conclusões e as implicações da política para futuras pesquisas.

\section{Modelo de Crescimento}

O modelo apresentado consiste em uma aplicação do modelo protótipo de Ramsey-Cass-Koopmans, adaptado à problemática em Alesina e Rodrik (1994) e Ortiz-Moctezuma et al. (2010). 


\subsection{Abordagem do Controle Ótimo com Melhorias na Infraestrutura}

Os pressupostos do modelo protótipo de Ramsey-Cass-Koopmans consistem nos mesmos apresentados em Barro e Sala-i-Martin (2004), Aghion e Howitt (2009) e Acemoglu (2009).

Assim, existe um conjunto de famílias que vivem infinitamente escolhendo um nível de consumo destinado a maximizar suas utilidades e sujeitas a uma restrição de acumulação do capital. No presente modelo, distinguem-se os fatores de produção em dois tipos: fator que pode ser acumulado (estoque de capital) e o fator que não pode ser acumulado (estoque de mão de obra). Nesse sentido, o crescimento é conduzido pela expansão no estoque de capital (equação básica de acumulação do capital) que, por sua vez, é determinada pelas decisões de consumo e poupança dos indivíduos. O crescimento é endogenamente determinado, tomando a função de produção da economia como sendo linearmente homogênea nos fatores de produção. Por simplicidade e sem muitas perdas de generalidade, iremos abstrair o progresso tecnológico do modelo.

Os gastos públicos servem para financiar a expansão e melhoria no estoque de infraestrutura da economia. ${ }^{1}$ Seguindo a mesma função de produção agregada em Alesina e Rodrik (1994), com apenas uma sutil restrição na função de produção, na qual normalizou-se a força de trabalho em uma unidade $(l=1)$ e tomaram-se as demais variáveis em unidade relativa à força de trabalho normalizada:

$$
y(t)=A f(k, G)=A k(t)^{\alpha} G^{1-\alpha} \quad \text { ou } y=A k^{\alpha} G^{1-\alpha}
$$

Conforme a equação $1, A$ corresponde ao parâmetro que captura o efeito da tecnologia, $k$ ao estoque de capital e $G$ aos investimentos governamentais destinados a expandir e à manutenção da infraestrutura da economia. Os subscritos $t$, referentes ao tempo, serão desconsiderados a fim de evitar notações muita carregadas nas variáveis. Cada investimento governamental aplicado cresce e melhora a infraestrutura local, representado pela variável $z$. Assim, pode-se dizer que a infraestrutura é uma função monotônica e crescente dos investimentos, $z=\vartheta(G)$ . Conforme Ortiz-Moctezuma et al. (2010), a dinâmica da infraestrutura no tempo é representada pela equação diferencial:

$$
\frac{d z}{d t} \equiv \dot{z}=g-\delta z
$$

1 Na compreensão do termo "infraestrutura", o seu conceito consiste nas diversas dimensões de bens e serviços coletivos, incluindo energia, água, transportes, telecomunicações, etc., que potencializam os recursos produtivos. Para maiores detalhes sobre este conceito, ver Button (1998) e Lin e Doemeland (2012). 
Conforme os autores, g corresponde à taxa de crescimento da economia e $\delta$ à taxa de depreciação no estoque da infraestrutura. Um importante destaque na equação 2 consiste no efeito do crescimento sobre o avanço da infraestrutura. ${ }^{2}$ Conforme a equação, a expansão do crescimento cria uma demanda por infraestrutura que reforça o crescimento nos períodos seguintes. Assim, da mesma forma que os investimentos em infraestrutura contribuem para um crescimento na produtividade, o mesmo gera um impacto de forma reversa acompanhado pela expansão por novos investimentos.

Outros estudos ${ }^{3}$ vêm corroborando com as contribuições de Ortiz-Moctezuma et al. (2010) demonstrando a necessidade de um maior aprofundamento sobre a relação entre as variáveis.

Reformulando Ortiz-Moctezuma et al. (2010), nem todo crescimento implica uma nova demanda por infraestrutura. Fedderke, Perkins e Luiz (2006) analisaram o impacto dos investimentos em infraestrutura no crescimento da África do Sul. Os resultados do estudo sugerem que o feedback do crescimento na expansão da infraestrutura, embora esta seja observada, indica um efeito menos expressivo ao se comparar com a contribuição da infraestrutura no crescimento.

Para capturar as evidências de Fedderke, Perkins e Luiz (2006) na equação dinâmica de Ortiz-Moctezuma et al. (2010), ${ }^{4}$ sugere-se a seguinte modificação:

$$
\dot{Z}=\mu g-\delta z
$$

A função- $\mu$ corresponde à resposta do crescimento na geração de demanda por infraestrutura. Pode-se imaginar que uma parte do crescimento que repercute no avanço da infraestrutura é uma função decrescente da riqueza acumulada. Tal aspecto é explicado pela hipótese da convergência, que afirma que nas economias de baixa renda a velocidade de crescimento apresenta ser maior do que nas eco-

2 Ao contrário da equação 2 , os investimentos em infraestrutura geram um número significativo de empregos no curto prazo, especialmente na construção civil. Além disto, os projetos de infraestrutura podem repercutir em diversas outras indústrias, que não apenas na construção civil ou manufaturas (LIN; DOEMELAND, 2012).

3 Segundo Button (1998), duas importantes escolas de pensamento tratam o efeito da relação crescimento-infraestrutura de maneiras distintas. A abordagem keynesiana considera que toda renda somente pode ser estimulada pela infraestrutura quando o crescimento induz sua expansão em primeiro lugar. Assim, o crescimento é um dos principais fatores que condicionam os avanços na infraestrutura. Em uma abordagem alternativa, a escola clássica considera os avanços da infraestrutura como um insumo produtivo, seguindo as contribuições de Barro (1990) e Alesina e Rodrik (1994).

4 Embora esta reformulação se apresente de forma simples na equação, sua contextualização na problemática proposta por Ortiz-Moctezuma et al. (2010) não consistiu no modelo de Ramsey-Cass-Koopmans. Além disto, uma modelagem mais aprofundada consideraria o efeito dinâmico da função- $\mu$ como dependente do nível de riqueza da economia. Esta sugestão, embora crível, não faz parte do escopo desta pesquisa sendo, portanto, um direcionamento para futuras pesquisas. 
nomias mais ricas. Isto sugere que as taxas de crescimento superiores vinculadas a uma necessidade relativamente maior nas economias de baixa renda poderiam explicar o fato de uma política direcionada à expansão da infraestrutura contribuir para uma rápida dinâmica econômica (AGHION; HOWITT, 2009). No presente modelo teórico será desconsiderada tal vinculação, ou, ao menos, apresentada no modelo empírico. Tal restrição não inviabiliza a contribuição e as assunções do modelo teórico.

Por último, tem-se a clássica equação de acumulação do capital:

$$
\frac{d k}{d t} \equiv \dot{k}=f(k, G)-c-d k
$$

Com base na equação 4, c corresponde ao nível de consumo, que é função do tempo, e $d$ à taxa de depreciação do capital, invariante ao tempo.

Assume-se que todos os indivíduos da economia apresentam preferências idênticas e que buscam maximizar a sua utilidade esperada ao longo do tempo. A função $u($.) representa a utilidade dos indivíduos, sendo crescente no argumento e côncava $-u^{\prime}()>$.0 e $u^{\prime \prime}()<$.0 . Logo, pode-se representar $u(c)$ por:

$$
u(c)=\frac{c^{1-\sigma}-1}{1-\sigma}
$$

Outros importantes pressupostos são definidos a seguir:

$$
\begin{aligned}
& k(0)=k_{o}>0 \\
& z(0)=z_{0}>0 \\
& c(0)=c_{0}>0 \\
& d>\delta
\end{aligned}
$$

O próximo passo consiste em obter uma solução ao problema dos indivíduos que buscam maximizar sua utilidade sujeita às restrições de acumulação do capital e de expansão da infraestrutura.

\subsection{Problema de Otimização}

O problema de otimização consiste na maximização da equação 5 ao longo de um horizonte infinito, descontando a utilidade por uma taxa de preferência temporal - $e^{-\rho t}$, e sujeita às restrições das equações 3-4 e P1-4.

$$
\begin{aligned}
& \underbrace{\text { máx }}_{c \geq 0} J[c, k, z, t] \stackrel{\text { def }}{=} \int_{0}^{+\infty} e^{-\rho t} \cdot u(c) \cdot d t
\end{aligned}
$$

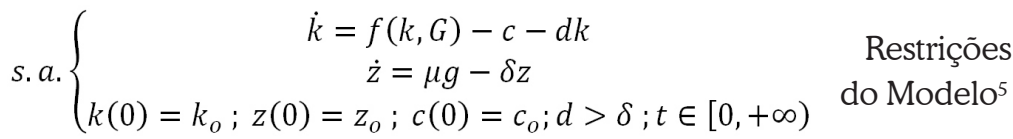

5 Outro pressuposto consiste nos conjuntos que definem as variáveis controle e de estado. Cada 
Para solucionar a equação 6, construiu-se a função hamiltoniana:

$$
\mathcal{H}\left[c, k, z, \lambda_{k}, \lambda_{z}, t\right]=u(c)+\lambda_{k}[f(k, G)-c-d k]+\lambda_{z}[\mu g-\delta z]
$$

Uma vez que a equação 7 satisfaz os critérios de otimalidade definidos em Caputo (2005) ${ }^{6}$ e Acemoglu (2009) ${ }^{7}$, então o Princípio do Máximo de Pontryagin garante as seguintes soluções:

$$
\dot{\lambda}_{k}=\rho \lambda_{k}-\frac{\partial \mathcal{H}}{\partial k} \text { e } \dot{\lambda}_{z}=\rho \lambda_{z}-\frac{\partial \mathcal{H}}{\partial z} \quad \frac{\partial \mathcal{H}}{\partial c}=0
$$

Com as condições de transversalidade:

$$
\lim _{t \rightarrow+\infty} e^{-\rho t} \lambda_{k} k=0 \operatorname{elim}_{t \rightarrow+\infty} e^{-\rho t} \lambda_{z} z=0
$$

As equações finais que satisfazem as soluções anteriores são dadas por:

$$
\begin{gathered}
u^{\prime}(c)-\lambda_{k}=0 \\
\dot{\lambda}_{z}=\rho \lambda_{z}-\left[f_{k}^{\prime}(k, G)-d\right] \mathrm{e} \dot{\lambda}_{z}=\rho \lambda_{z}-\left[-\lambda_{z} \delta\right]
\end{gathered}
$$

A seguir, os valores que satisfazem os critérios de solução:

$$
\begin{gathered}
\frac{\dot{c}}{c}=\frac{1}{\sigma}\left[f_{k}^{\prime}(k, G)-\rho-d\right] \\
\frac{\dot{\lambda}_{z}}{\lambda_{z}}=\rho+\delta
\end{gathered}
$$

As condições de estado estacionário implicam $\lim _{t \rightarrow+\infty}\left(\frac{\dot{c}}{c}, \frac{\dot{\lambda}_{z}}{\lambda_{z}}\right)=0$ :

$$
\begin{gathered}
f_{k}^{\prime}(\hat{k}, G)-\rho-d=0 \Leftrightarrow \alpha \hat{k}^{\alpha-1} G^{1-\alpha}=\rho+d \\
\rho+\delta=0 \Leftrightarrow \rho=-\delta \\
\hat{k}=\left[\frac{\alpha}{d-\delta}\right]^{\frac{1}{1-\alpha}} G
\end{gathered}
$$

A solução do problema mostra que os investimentos em infraestrutura aumentam o estoque de capital de longo prazo, estimulando o crescimento. Por sua

conjunto depende do tempo que por simplicidade será abstraído desta análise. Neste caso, iremos considerar que cada conjunto seja aberto e limitado, para restringir as possíveis soluções de fronteira. Logo, $c, k, z \in \operatorname{int}\{\mho \times K \times Z\}$, de forma que $\mathrm{c}:[0,+\infty) \rightarrow \mho ; \mathrm{k}:[0,+\infty) \rightarrow \mathrm{K} ; \mathrm{z}:[0,+\infty) \rightarrow Z$. Além destes pressupostos, os multiplicadores dinâmicos da função hamiltoniana são definidos como: $\lambda_{\mathrm{k}}:[0,+\infty) \rightarrow \mathbb{R}_{+}$e $\lambda_{\mathrm{z}}:[0,+\infty) \rightarrow \mathbb{R}_{\boldsymbol{+}}$. Para evitar um carregamento nas notações, tais pressupostos foram inseridos nesta nota de rodapé.

6 Ver Teorema 14.1.

$7 \quad$ Ver Lema 7.1, Teorema 7.9 e Teorema 7.10. 
vez, o crescimento reforça a demanda por infraestrutura que novamente repercute em um novo crescimento (satisfazendo a equação 3).

\subsection{Incluindo a Tecnologia}

Na subseção anterior, considerou-se o parâmetro que captura o efeito da tecnologia normalizado e sendo igual a uma unidade $(A=1)$. Nesta subseção, será apresentada a influência da tecnologia na produção e nas soluções de estado estacionário. Embora novamente seja abstraído o efeito do progresso tecnológico (da taxa de progresso), destaca-se o fator condicionante que a tecnologia oferece para as soluções de estado estacionário. Um bom exercício alternativo consiste em considerar um modelo mais abrangente, contudo, isso não compromete a generalidade aqui apresentada.

O problema da equação 6 agora sofre uma pequena modificação na função de produção, incluindo o parâmetro tecnológico:

$$
\mathcal{H}\left[c, k, z, \lambda_{k}, \lambda_{z}, t\right]=u(c)+\lambda_{k}[A f(k, G)-c-d k]+\lambda_{z}[\mu g-\delta z]
$$

A solução de estado estacionário do novo problema sujeito às mesmas restrições é dada por:

$$
\begin{aligned}
A f_{k}^{\prime}(\hat{k}, G)-\rho-d & =0 \Leftrightarrow \alpha A \hat{k}^{\alpha-1} G^{1-\alpha}=\rho+d \\
\rho+\delta & =0 \Leftrightarrow \rho=-\delta \\
\hat{k} & =\left[\frac{\alpha}{d-\delta}\right]^{\frac{1}{1-\alpha}} G
\end{aligned}
$$

A solução apresentada mostra que a tecnologia afeta positivamente o estoque de capital, assim como os gastos em infraestrutura. Contudo, tais investimentos concorrem com outras políticas, de forma que a distribuição dos recursos é afetada pelo ritmo da atividade econômica.

Nas economias menos desenvolvidas, o custo de oportunidade de muitas atividades intensivas em inovação pode apresentar um alto valor. Aghion e Howitt (2009) apresentaram importantes evidências empíricas demonstrando que, nas economias menos desenvolvidas, as atividades intensivas na adoção ou difusão de tecnologias provenientes das economias desenvolvidas podem oferecer uma velocidade de crescimento superior em relação às atividades intensivas em inovação.

Nessa linha de raciocínio, Aghion, García-Peñalosa e Howitt(2004) mostraram que uma infraestrutura adequada ao crescimento pode estimular a inovação e, especialmente, a difusão de tecnologias da fronteira. Os autores argumentaram que um sistema de telecomunicações desenvolvido e uma boa infraestrutura viária e de transportes podem aumentar a mobilidade da mão de obra qualifica- 
da entre as firmas e as indústrias. Assim, esse cenário pode resultar em uma alta taxa de inovação e difusão tecnológica em vários setores da economia, na medida em que a economia vai se aproximando da fronteira tecnológica. Outros estudos vêm apontando para resultados similares, destacando-se Lucas (1993), Aghion e Schankerman(1999) e Acemoglu, Aghion e Zilibotti (2006).

\subsection{Dualidade da Tecnologia e dos Investimentos Governamentais}

Em uma importante contribuição, a partir do estudo de Acemoglu, Aghion e Zilibotti (2006), a tecnologia pela ótica da empresa é percebida em dois importantes componentes que dependem de estratégias bem distintas: (1) um componente ligado diretamente às atividades intensivas em inovação e (2) outro componente ligado diretamente às atividades menos intensivas em inovação e mais intensivas na difusão e implementação de tecnologias existentes na fronteira.

Conforme os autores, uma simples adaptação pode ser vista da seguinte forma:

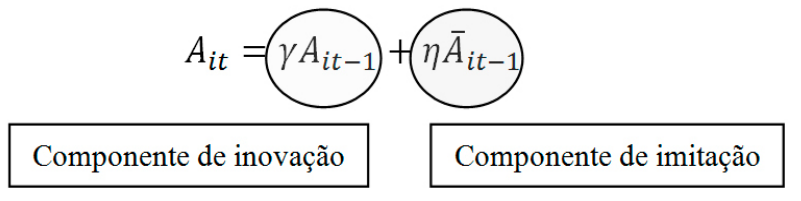

Segundo a equação 9, a produtividade da economia i no período $t$ depende do avanço associado às atividades inovativas ( $\left.\gamma A_{i t-1} \therefore \gamma>1\right)$, que melhoram a produtividade no período seguinte, mais um processo atribuído à difusão de tecnologias da fronteira $\left(\eta \bar{A}_{i t-1} \therefore A_{i t-1} \leq \bar{A}_{i t-1 ;} ; \forall \eta \in(0,1)\right)$. De acordo com Acemoglu, Aghion e Zilibotti (2006), esta dualidade impõe restrições na política, de forma que a contribuição dos investimentos no processo de convergência depende de uma adequada alocação dos recursos, conforme o estágio de desenvolvimento da economia (ou proximidade com a fronteira).Tal restrição pode ser visualizada quando dividimos a equação 9 pela produtividade da fronteira $\left(\bar{A}_{i t}\right)$.

Supondo que a fronteira cresce a uma taxa exógena e constante $\bar{g}$, tem-se:

$$
\begin{gathered}
\frac{A_{i t}}{\bar{A}_{i t}}=\frac{\gamma A_{i t-1}}{\bar{A}_{i t}}+\frac{\eta \bar{A}_{i t-1}}{\bar{A}_{i t}} \therefore \bar{A}_{i t}=(1+\bar{g}) \bar{A}_{i t-1} \\
a_{i t}=(1+\bar{g})^{-1}\left[\gamma a_{i t-1}+\eta\right]
\end{gathered}
$$

Na equação 10, os parâmetros $\gamma, \eta$ refletem diretamente os arranjos institucionais de uma política que afeta os esforços em inovação e imitação. Cada parâmetro está limitado em um valor máximo e mínimo, $\gamma \in[\underline{\gamma}, \bar{\gamma}] ; \eta \in[\underline{\eta}, \bar{\eta}]$. Assim, existem dois importantes cenários institucionais: i) instituições com elevada ênfase no esforço em inovação e baixa ênfase na imitação, P1: $(\bar{\gamma}, \underline{\eta})$ e ii) instituicões com elevada ênfase no esforço em imitação e baixa ênfase na inovação, P2: $(\underline{\gamma}, \bar{\eta})$. 
Dada a restrição orçamentária do governo, a composição dos recursos destinados a uma rápida velocidade de crescimento depende do quão próxima ou distante se encontra uma determinada economia em relação à fronteira. Nesse sentido, as economias mais afastadas da fronteira desfrutam de uma precária infraestrutura, de maneira que os investimentos para tal finalidade podem repercutir em uma maior contribuição no crescimento. À medida que a economia vai se aproximando da fronteira, os recursos destinados à inovação vão apresentando uma importância relativamente maior, uma vez que tais investimentos são responsáveis pela aproximação na vizinhança e no próprio deslocamento da fronteira. Recentes evidências dão suporte a esta dualidade, destacando-se Griffith, Redding e Van Reenen (2004), Caselli e Coleman (2006), Wu (2010).Cada cenário institucional é importante à medida que incorporamos as diferenças tecnológicas e de desenvolvimento entre as economias. Embora os esforços inovativos garantam uma convergência para a fronteira, esta velocidade de aproximação pode ser potencializada inicialmente com importantes investimentos em infraestrutura, criando oportunidades para o capital e reduzindo os custos associados à deficiência da infraestrutura (QUAH, 1996).

A seguir, será apresentado o modelo econométrico que se destina a operacionalizar a relação entre as variáveis de forma a testar os questionamentos levantados no modelo teórico.

\section{Abordagem Empírica}

Nesta seção é apresentado o procedimento metodológico adotado na realização do estudo. Inicialmente, faz-se a descrição do painel de dados analisados e, em seguida, do modelo econométrico empregado na estimação dos impactos.

\subsection{Definição da Amostra e Operacionalização das Variáveis}

Para testar a importância dos investimentos públicos em infraestrutura será considerada uma amostra das 27 unidades federativas ao longo dos anos 2000 a 2011. Dessa forma, a natureza dos dados em forma de painel compreende um total de 324 observações. Os investimentos que abrangem a política de infraestrutura foram selecionados conforme cinco categorias: (1) Segurança Pública; (2) Energia; (3) Habitação e Urbanismo; (4) Saúde e Saneamento e (5) Transportes.

Conforme Montes e Reis (2011) estas dimensões imperam o importante comportamento da política de infraestrutura nos estados brasileiros. Os dados referentes a estas variáveis foram extraídos da Secretaria do Tesouro Nacional que fornece informações das despesas por função nas diferentes unidades federativas. Outra despesa importante e que foi empregada no modelo consiste na despesa 
em Educação e Cultura. Sua desagregação na composição dos investimentos em infraestrutura consiste em isolar o efeito da política de educação no crescimento dos estados.

Para medir o impacto sobre a riqueza, utilizou-se a medida do PIB per capita disponibilizado pelo Instituto Brasileiro de Geografia e Estatística (IBGE). As variáveis que capturam a dinâmica de aproximação com a fronteira consistem nos investimentos em Ciência $\mathcal{E}$ Tecnologia ${ }^{8}$ e na formação da mão de obra com alta qualificação, compreendendo o total de concluintes no ensino superior. Tais informações foram obtidas do Ministério da Ciência, Tecnologia $\mathcal{E}$ Inovação (MCTI). Por último, um importante componente do estoque de capital humano de cada estado consiste nos anos de escolaridade média, para uma população de 25 anos ou mais, extraídos dos Microdados da Pesquisa Nacional por Amostra de Domicílios (PNAD), do IBGE.

\subsection{Modelo Econométrico}

A equação a ser estimada consiste em:

$$
\begin{gathered}
\log \left(\frac{Y}{L}\right)_{i t}=\alpha+\delta_{j}+\gamma_{t}+\beta_{1} \log (G)_{i t-1}+\beta_{2} \log \left(L_{S}\right)_{i t-1}+\beta_{2} \log (C T)_{i t-1}+\beta_{2} \log (E)_{i t}+ \\
\beta_{2} \log (h)_{i t-1}+\varepsilon_{i t}
\end{gathered}
$$

ME.1

Conforme a equação a ser estimada ME.1, a variável dependente $\left(\frac{Y}{L}\right)$ corresponde ao PIB per capita do estado i no tempo t. Os gastos em infraestrutura são capturados pela variável $G$, considerando uma defasagem de um ano (t-1), destinado a vislumbrar o efeito dos investimentos no período seguinte. As demais variáveis $L_{S}, C T, E, h$ correspondem, respectivamente, ao número de concluintes no ensino superior, aos gastos em Ciência $\mathcal{Z}$ Tecnologia, aos investimentos estaduais em Educação Básica e Cultura e aos anos de escolaridade média da população.

Os efeitos fixos associados à heterogeneidade das regiões e aos choques de natureza temporal, e comuns aos estados e às regiões, são capturados respectivamente pelos parâmetros a serem estimados $\delta_{j}, \gamma_{t} \cdot{ }^{9}$ Por último, tem-se a perturbação estocástica que compreende todos os demais fatores que não estão sob o controle do modelo estatístico, $\varepsilon_{i t} \sim N\left(0, \sigma_{\varepsilon}^{2}\right)$.

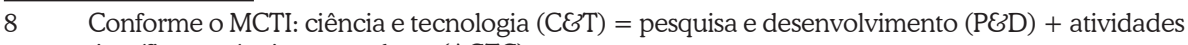
científicas e técnicas correlatas (ACTC).

9 Os efeitos fixos associados aos estados foram agregados aos efeitos "regionais" de forma a não comprometer os graus de liberdade de modelo. Um número excessivo de parâmetros estimados reduz consideravelmente os graus de liberdade. 


\subsubsection{Método de Estimação}

Para capturar o efeito dos investimentos considerando diferentes contextos de desenvolvimento, o método de regressão com efeitos fixos não consiste em uma metodologia apropriada. Isso porque tal método aborda um resumo das médias das distribuições associadas ao conjunto observado de variáveis. Assim, uma média consiste em uma medida de tendência central para todas as observações. Ao contrário disto, poderíamos considerar diversas outras médias associadas às partes percentuais da distribuição, compreendendo uma visão mais completa do conjunto de dados e das relações entre as variáveis (KOENKER, 2005).

O método que permite vislumbrar tal procedimento consiste na abordagem de regressão quantílica, em homenagem às contribuições de Koenker e Bassett (1978). A solução no problema de estimar regressões em diferentes quantis consiste na seguinte forma:

$$
y_{i t}=x_{i t}^{\prime} \beta(\tau)+\varepsilon_{i t}(\tau) \quad \text { ME. } 2
$$

onde a função $Q_{y}(\tau \mid \boldsymbol{x})=\boldsymbol{x}_{i t}^{\prime} \beta(\tau)$ representa a função condicional do $\tau$-ésimo quantil, onde $\tau \epsilon(0,1)$. Desta forma, precisa-se encontrar os parâmetros que minimizam o problema de otimização:

$$
\begin{gathered}
\min _{\beta(\tau) \in \mathbb{R}^{p}} \frac{1}{N}\left\{\sum_{i, t: y_{i t} \geq x_{i t}^{\prime} \beta} \tau\left|y_{i t}-x_{i t}^{\prime} \beta\right|+\sum_{i, t: y_{i t}<x_{i t}^{\prime} \beta}(1-\tau)\left|y_{i t}-x_{i t}^{\prime} \beta\right|\right\}=\quad \text { ME. } 3 \\
\min _{\beta(\tau) \in \mathbb{R}^{p}} \frac{1}{N} \sum \rho(\tau) \varepsilon_{i t}
\end{gathered}
$$

Conforme a equação 3, a função $\rho(\tau)$ consiste em uma função linear piecewise, que divide a distribuição conforme o $\tau$-ésimo quantil. A função $\rho(\tau \mid \varepsilon)$ é definida conforme a restrição:

$$
\rho(\tau \mid \varepsilon)= \begin{cases}\tau \varepsilon_{i t} & , \text { if } \varepsilon_{i t} \geq 0 \\ (\tau-1) \varepsilon_{i t} & , \text { if } \varepsilon_{i t}<0\end{cases}
$$

A solução da equação ME. 3 envolve um procedimento de programação linear, disponível em vários pacotes estatísticos de regressão linear. Dada a sutileza do método, recentes pesquisas vêm aplicando tal abordagem como forma de considerar diferentes relações entre as variáveis econômicas dentro do mesmo conjunto de observações. Tais estudos vêm aplicando o método, seja em questões microeconômicas (COAD, 2011; COAD; RAO, 2011; COAD; RAO, 2006) seja em modelos macroeconômicos (KOENKER; HALLOCK, 2001; BUCHINSKY, 1994). 


\subsubsection{Eficiência no Método de Regressão Quantllica}

$\mathrm{Na}$ existência de erros não normais e na presença de pontos discrepantes na amostra (outliers), o método tradicional de mínimos quadrados ordinários é ineficiente, embora seja consistente caso o pressuposto de ortogonalidade dos erros não seja violado. Neste caso, a confiabilidade das estimativas fica comprometida, uma vez que os intervalos de confiança apresentam claros sinais de tendenciosidade (KOENKER; HALLOCK, 2001).

Neste caso, o método de regressão quantílica apresenta uma forma mais apropriada de tratar a relação entre as variáveis sob tais circunstâncias. Ainda que tal método seja mais robusto na presença de tais anomalias dos dados, sua persistência pode comprometer a confiança nas estimativas (KOENKER, 2005).

Neste caso, um importante procedimento vem sendo aplicado como forma de correção quando o método apresenta sinais de violação nos pressupostos de normalidade. Este procedimento, graças às contribuições de Efron (1979), emprega um método de reamostragem que calcula diferentes estimativas de parâmetros, procedendo com uma média das B-ésimas estimativas amostrais. Tal procedimento é conhecido como Método de Bootstrap. A maior vantagem destametodologia consiste nas suposições de normalidade dos resíduos que não são requisitadas para o emprego do método, garantindo estimativas consistentes da variância dos parâmetros. Essa técnica vem apresentando esta e muitas outras vantagens com bastante frequência na literatura econométrica (ver recentemente Greene (2012) e Cameron e Trivedi (2005)).

Embora essa técnica seja bastante sofisticada, o número de replicações da amostra tem sido algo de muita controvérsia no debate acadêmico. Por exemplo, Efron e Tibshirani (1993) afirmam que 50 replicações são um número suficiente para se obter uma boa estimativa de erro-padrão. Contudo, Andrews e Buchinsky (2001) sugerem um tamanho maior de replicações para obter estimativas mais confiáveis, adotando um número $B=400$. Na presente pesquisa, será aplicado o procedimento apresentado por Andrews e Buchinsky (2001), uma vez que um número maior de replicações permite uma estimativa mais consistente da média dos parâmetros.

\section{Resultados e Discussão}

A seguir, apresentam-se o resultado dos modelos estimados e a discussão com pesquisas recentes na mesma temática. 


\subsection{Análise dos Resultados}

A equação ME.1 foi estimada considerando três importantes métodos: Mínimos Quadrados Ordinários Agrupados (Pooled), Mínimos Quadrados Ordinários com Variáveis Dummy (Efeitos Fixos) e Regressão Quantílica com Efeitos Fixos. A Tabela 1 apresenta os resultados referentes aos dois primeiros métodos. O método de MQO apresentou significância estatística em quase todos os parâmetros, com exceção dos gastos em CETT. Um aumento nos gastos em infraestrutura contribui para um crescimento médio no PIB per capita no período seguinte de aproximadamente $0,143 \%$ (significativo a 1\%). Com relação ao estoque de mão de obra com qualificação (concluintes do ensino superior), o resultado esperado é coerente com a teoria. Desta forma, $1 \%$ no aumento de formandos no ensino superior contribui para um crescimento no PIB per capita do período seguinte de aproximadamente $0,608 \%$ (significativo a $1 \%$ ). Os investimentos realizados pelos estados em educação e cultura apresentam uma relação positiva com o PIB per capita, de forma que $1 \%$ no aumento destes investimentos contribui para um crescimento médio de aproximadamente $0,328 \%$.

Por último, tem-se a variável referente aos anos de escolaridade média da população, usualmente empregada como estoque agregado de capital humano. Um aumento de $1 \%$ na escolaridade contribui para um crescimento médio de $0,101 \%$ no PIB per capita. O poder de explicação do modelo apresentou-se elevado, de forma que as variáveis ajudam a explicar $96,80 \%$ das variações na variável dependente.

Tabela 1 - Resultados da estimação da equação ME1 por mínimos quadrados ordinários agrupados (pooled), mínimos quadrados ordinários com variáveis dummy (efeitos fixos)

\begin{tabular}{c|cccc}
\hline \multirow{3}{*}{ Variável } & $(\mathbf{1})$ & $\mathbf{( 2 )}$ & $\mathbf{( 3 )}$ & $\mathbf{( 4 )}$ \\
\cline { 2 - 5 } & $\begin{array}{c}\text { OLS Agru- } \\
\text { pado }\end{array}$ & Ef. Fixos & Ef. Fixos & Ef. Fixos \\
\hline $\log (\mathrm{G})_{\mathrm{t}-1}$ & $0.143 * * *$ & $0.234 * * *$ & $0.267 * * *$ & $0.318^{* * *}$ \\
& $(0.0532)$ & $(0.0528)$ & $(0.0618)$ & $(0.0611)$ \\
$\log \left(\mathrm{L}_{\mathrm{S}}\right)_{\mathrm{t}-1}$ & $0.608 * * *$ & $0.611 * * *$ & $0.449 * * *$ & $0.501 * * *$ \\
& $(0.0370)$ & $(0.0331)$ & $(0.0496)$ & $(0.0522)$ \\
$\log (\mathrm{CT})_{\mathrm{t}-1}$ & 0.00733 & $0.0228 *$ & 0.00677 & 0.0175 \\
& $(0.0150)$ & $(0.0130)$ & $(0.0144)$ & $(0.0131)$ \\
$\log (\mathrm{E})_{\mathrm{t}}$ & $0.328 * * *$ & $0.235^{* * *}$ & $0.340 * * *$ & $0.254 * * *$ \\
& $(0.0594)$ & $(0.0542)$ & $(0.0509)$ & $(0.0515)$ \\
\hline
\end{tabular}


conclusão.

\begin{tabular}{c|cccc}
\hline \multirow{2}{*}{ Variável } & $(\mathbf{1})$ & $\mathbf{( 2 )}$ & $\mathbf{( 3 )}$ & $\mathbf{( 4 )}$ \\
\cline { 2 - 5 } & $\begin{array}{c}\text { OLS Agru- } \\
\text { pado }\end{array}$ & Ef. Fixos & Ef. Fixos & Ef. Fixos \\
\hline $\log (\mathrm{h})_{\mathrm{t}-1}$ & $\begin{array}{c}0.101^{* * *} \\
(0.0176)\end{array}$ & $\begin{array}{c}0.120^{* * *} \\
(0.0159)\end{array}$ & $\begin{array}{c}0.0422 \\
(0.0273)\end{array}$ & $\begin{array}{c}0.111^{* * *} \\
(0.0278)\end{array}$ \\
\hline $\begin{array}{c}\text { Efeitos fixos- } \\
\text { ano }\end{array}$ & $\mathrm{NÃO}$ & $\mathrm{SIM}$ & $\mathrm{NÃO}$ & $\mathrm{SIM}$ \\
$\begin{array}{c}\text { Efeitos fixos- } \\
\text { região }\end{array}$ & $\mathrm{NÃO}$ & $\mathrm{NÃO}$ & $\mathrm{SIM}$ & $\mathrm{SIM}$ \\
\hline $\begin{array}{c}\mathrm{N}^{\circ} \\
\text { Observações }\end{array}$ & 324 & 324 & 324 & 324 \\
$\mathrm{R}^{2}$ & 0.968 & 0.973 & 0.972 & 0.976 \\
Wald chi $^{2}$ & 10001 & 9763 & 15970 & 13491 \\
Prob> chi $^{2}$ & 0.000 & 0.000 & 0.000 & 0.000 \\
\hline
\end{tabular}

Fonte: Elaboração própria.

Nota: A variável dependente consiste no $\log (\mathrm{PIB}$ per capita). As estimativas de erro-padrão foram calculadas a partir do método de Bootstrap com 400 replicações amostrais. Os asteriscos correspondem aos respectivos níveis de significância, ${ }^{* *} \mathrm{p}<0.01,{ }^{* *} \mathrm{p}<0.05,{ }^{*} \mathrm{p}<0.1$.

Com a inclusão dos efeitos fixos relativos ao tempo, coluna (2), percebem-se algumas variações nos parâmetros estimados. A elasticidade dos gastos em infraestrutura apresentou um aumento expressivo de aproximadamente $64 \%$ em relação ao método agrupado (significativo a 1\%). Desta forma, espera-se que um aumento de $1 \%$ nos gastos gere um crescimento médio de $0,234 \%$ no PIB per capita. Todas as demais variáveis, com exceção dos gastos em educação e cultura, apresentaram um aumento em relação ao método agrupado, são elas: (i) concluintes no ensino superior, uma variação positiva de $0,49 \%$ (parâmetro significativo a 1\%); (ii) gastos em CẺT, uma variação positiva de $211,05 \%$ (parâmetro significativo a 1\%) e (iii) anos de escolaridade, uma variação positiva de $18,81 \%$ (parâmetro significativo a 1\%). O poder de explicação aumentou para $97,30 \%$.

$\mathrm{Na}$ coluna (3) e (4), a elasticidade dos gastos em infraestrutura apresentou uma tendência de crescimento em relação ao método agrupado, com uma variação positiva de $86,71 \%$ (coluna (3)) e 122,38\% (coluna (4)). Em cada caso, os parâmetros apresentaram-se significativos a 1\%. Com a inclusão dos efeitos fixos regionais (coluna (3)), observou-se uma queda na elasticidade dos concluintes do ensino superior, com $0,449 \%$ contra $0,611 \%$, na coluna (2). Embora o parâmetro tenha apresentado significância estatística, percebe-se uma clara sensibilidade com a inclusão dos efeitos fixos regionais. A tendência de aumento na elasticida- 
de é retomada na coluna (4) quando incluímos os efeitos de tempo, juntamente com os efeitos regionais (significativo a 1\%). A elasticidade dos gastos em CETT apresentou uma queda com a exclusão dos efeitos de tempo e inclusão dos efeitos regionais (coluna (3)), embora apresente um crescimento com a junção de ambos os efeitos (coluna (4)). Em cada caso, o parâmetro apresentou rejeitar sinais de significância estatística.

Não obstante tenha se observado uma redução no impacto dos investimentos em CEST, os efeitos regionais apresentaram uma influência no aumento da elasticidade dos gastos em educação e cultura. Na coluna (3), o parâmetro calculado apresentou uma variação positiva de 3,66\% em relação ao método agrupado (significativo a 1\%). Após a inclusão dos efeitos fixos relativos ao tempo, observa-se novamente uma queda no parâmetro, fazendo-o gravitar próximo ao parâmetro calculado na coluna (2). Esta sensibilidade também é observada no parâmetro da variável anos de escolaridade.

A exclusão dos efeitos de tempo, ainda que controlados pelos efeitos regionais, reduziu a estimativa em $58,22 \%$, embora tenha apresentado rejeitar sinais de significância estatística. Após a inclusão dos efeitos de tempo (coluna (4)), o parâmetro estimado apresentou um crescimento de $9,90 \%$ em relação ao método agrupado. Tais distorções mostram que os diferentes controles na amostra exercem influência significativa nos parâmetros estimados.

Além da influência observada, o poder de explicação do modelo vem apresentando um crescimento a partir da inclusão dos efeitos fixos, ainda que tal crescimento tenha se comportado de forma suave. Agregando os efeitos de tempo e regionais, as variáveis do modelo ajudam a explicar 97,60\% (coluna (4)) contra $97,20 \%$ quando excluímos os efeitos de tempo e consideramos apenas os efeitos regionais (coluna (3)).

A Tabela 2 apresenta os resultados a partir da regressão quantílica estimada, considerando um número de réplicas de bootstrap $B=400$. Os coeficientes da regressão quantílica podem ser interpretados como uma derivada parcial do quantil condicional da variável dependente (PIB per capita) com relação a um regressor particular, $\frac{\partial Q_{y}(\tau \mid x)}{\partial x_{i t}^{\prime}}$. Nesse sentido, o parâmetro estimado é interpretado como uma mudança marginal na variável dependente, dado o $\tau$-ésimo quantil condicional, em decorrência da mudança marginal. Os resultados mostram um declínio no parâmetro associado aos investimentos em infraestrutura. Isto indica que o efeito deste investimento como crescimento no PIB per capita apresenta um comportamento distinto nos diferentes quantis condicionais. 
Tabela 2 - Resultados da regressão quantílica

\begin{tabular}{|c|c|c|c|c|}
\hline \multirow{3}{*}{ Variável } & \multicolumn{4}{|c|}{ Regressão Quantílica (\%) } \\
\hline & (1) & (2) & (3) & (4) \\
\hline & q25 & q50 & q75 & q90 \\
\hline \multirow[t]{2}{*}{$\log (G)_{t-1}$} & $0.522 * * *$ & $0.351 * * *$ & $0.208^{* *}$ & $0.232 * * *$ \\
\hline & $(0.0642)$ & $(0.0893)$ & $(0.102)$ & $(0.0807)$ \\
\hline \multirow[t]{2}{*}{$\log \left(\mathrm{L}_{\mathrm{S}}\right)_{\mathrm{t}-1}$} & $0.353 * * *$ & $0.499 * * *$ & $0.589 * * *$ & $0.557 * * *$ \\
\hline & $(0.0914)$ & $(0.0660)$ & $(0.0733)$ & $(0.0761)$ \\
\hline \multirow[t]{2}{*}{$\log (\mathrm{CT})_{\mathrm{t}-1}$} & 0.00587 & 0.0108 & 0.0127 & $0.0419 * *$ \\
\hline & $(0.0160)$ & $(0.0142)$ & $(0.0159)$ & $(0.0186)$ \\
\hline \multirow[t]{2}{*}{$\log (E)_{t-1}$} & $0.225 * * *$ & $0.207 * * *$ & $0.251 * * *$ & $0.250 * * *$ \\
\hline & $(0.0717)$ & $(0.0654)$ & $(0.0643)$ & $(0.0578)$ \\
\hline \multirow[t]{2}{*}{$\log (h)_{t-1}$} & $0.0872 * *$ & $0.124 * * *$ & $0.114 * * *$ & $0.0923 * *$ \\
\hline & $(0.0365)$ & $(0.0386)$ & $(0.0409)$ & $(0.0401)$ \\
\hline $\begin{array}{c}\text { Efeitos fixos- } \\
\text {-ano }\end{array}$ & SIM & SIM & SIM & SIM \\
\hline $\begin{array}{l}\text { Efeitos fixos- } \\
\text {-região }\end{array}$ & SIM & SIM & SIM & SIM \\
\hline $\mathrm{N}^{0}$ Observações & 324 & 324 & 324 & 324 \\
\hline Pseudo-R2 & 0.8576 & 0.8552 & 0.8598 & 0.8756 \\
\hline
\end{tabular}

Fonte: Elaboração própria.

Nota: A variável dependente consiste no log (PIB per capita). As estimativas de erro-padrão foram calculadas a partir do método de bootstrap com 400 replicações amostrais. Os asteriscos correspondem aos respectivos níveis de significância, ${ }^{* * *} \mathrm{p}<0.01,{ }^{* *} \mathrm{p}<0.05,{ }^{*} \mathrm{p}<0.1$.

Desta forma, este comportamento atribuído pelo tamanho do efeito depende da localização na distribuição condicional. Nos quantis inferiores, caracterizados por uma restrição no PIB per capita, o impacto do investimento é superior e vai declinando à medida que relaxamos o tamanho do quantil. Embora os resultados apresentados na Tabela 1 tenham demonstrado um impacto médio positivo destes recursos, nos quantis condicionais mais baixos os parâmetros estimados são superiores, ao contrário dos quantis condicionais mais elevados, indicando uma notória tendência de inversão à média.

Ao longo dos quantis condicionais, os parâmetros estimados apresentaram uma elevada significância estatística. A partir disto, para os estados com renda limitada nos quantis condicionais inferiores, a elasticidade dos investimentos em infraestrutura é maior. Isto sugere que os investimentos em infraestrutura têm im- 
portância relativamente maior nos estados de baixa renda ou, pelo menos, contribui para um crescimento notoriamente maior nestes estados.

A tendência na queda do parâmetro é visivelmente observada em uma condição superior ao parâmetro estimado por efeitos fixos, coluna (4). A partir do quantil 0,75, percebe-se um suave crescimento no parâmetro com uma tendência de estabilizar-se o efeito. Este comportamento pode ser atribuído ao rápido crescimento que pode ser desfrutado pelas economias de baixa renda, criando novas demandas por infraestrutura e reforçando o crescimento, como apresentado no modelo teórico.

Na Figura 1, têm-se as elasticidades dos gastos em CETT por quantil condicional. Ao contrário dos gastos em infraestrutura, a elasticidade dos gastos em CEST apresenta uma clara reversão à média. Nos quantis condicionais inferiores, a elasticidade parcial deste investimento é inferior em relação à média obtida por efeitos fixos. Este padrão vai aumentando à medida que os quantis condicionais vão aumentando. Isto demonstra que nos quantis menores, em que a renda per capita é baixa, a contribuição em decorrência do aumento dos investimentos em CET é inferior à média obtida pelo método de efeitos fixos.

Figura 1 - Elasticidades dos gastos em CETT por quantil condicional

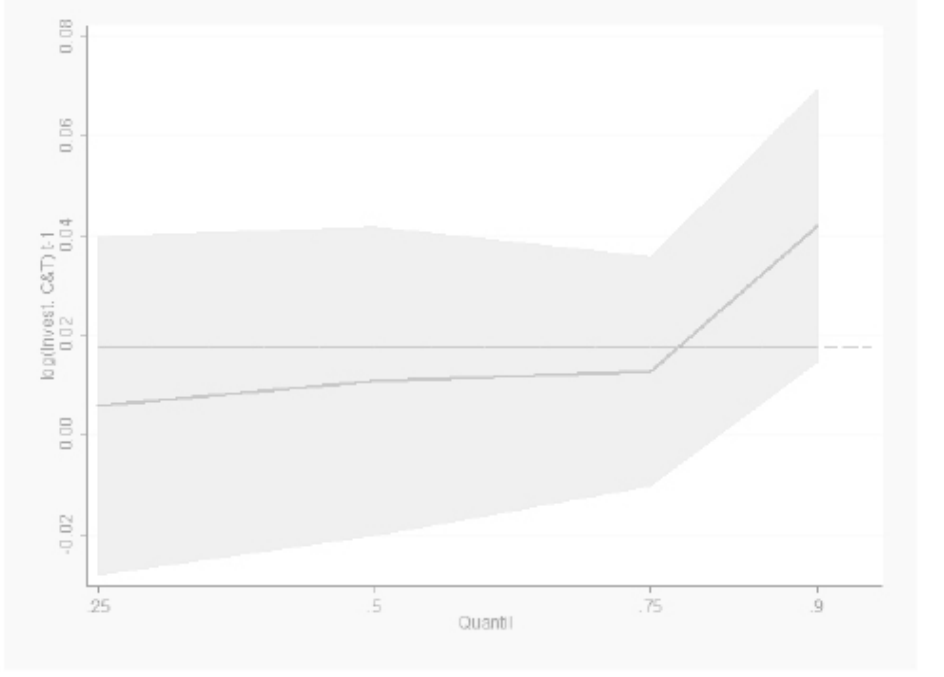

Fonte: Elaboração própria.

Desta forma, o comportamento atribuído pelo tamanho do efeito é afetado pela localização na distribuição condicional, em outras palavras, em estados com renda baixa, o efeito do investimento é inferior em relação aos estados com renda elevada. 
Na medida em que os quantis condicionais aumentam, a elasticidade parcial reverte-se à média chegando a atingir um valor superior em relação ao parâmetro médio por efeitos fixos. A exemplo disto, no quantil de $90 \%$ o parâmetro estimado está 139\% acima do parâmetro estimado por efeitos fixos. Considerando a mediana, esta diferença alcança um valor aproximadamente 4 vezes maior comparando ambos os parâmetros. Além disto, a significância do parâmetro é apresentada somente no quantil de $90 \%$, sendo não significativo nos quantis inferiores a este.

Este padrão diferenciado entre os quantis também é observado nas demais variáveis do modelo. Considerando os anos de escolaridade, no quantil inferior de $25 \%$ a elasticidade parcial encontra-se abaixo da mediana em aproximadamente $30 \%$. Esta diferença vai-se revertendo em direção à média, atingindo um valor superior nos quantis 50\% e 75\% (significativos a 1\%).

A Figura 2 mostra a evolução da elasticidade dos concluintes no ensino superior nos diferentes quantis condicionais. Assim como nos investimentos em CETT, o efeito do aumento proporcional deste regressor gera impactos diferentes. Nos estados com baixa renda, situados nos quantis abaixo da mediana, o aumento de $1 \%$ no número de concluintes no ensino superior contribui para um crescimento médio de $0,353 \%$ no PIB per capita. Este impacto aumenta quando consideramos o quantil da mediana, aproximadamente $0,499 \%$. Para os estados com renda maior, no quantis de $75 \%$ e $90 \%$, a elasticidade aumenta para $0,589 \%$ e $0,557 \%$, respectivamente. 
Figura 2 - Evolução da elasticidade dos concluintes no ensino superior nos diferentes quantis condicionais

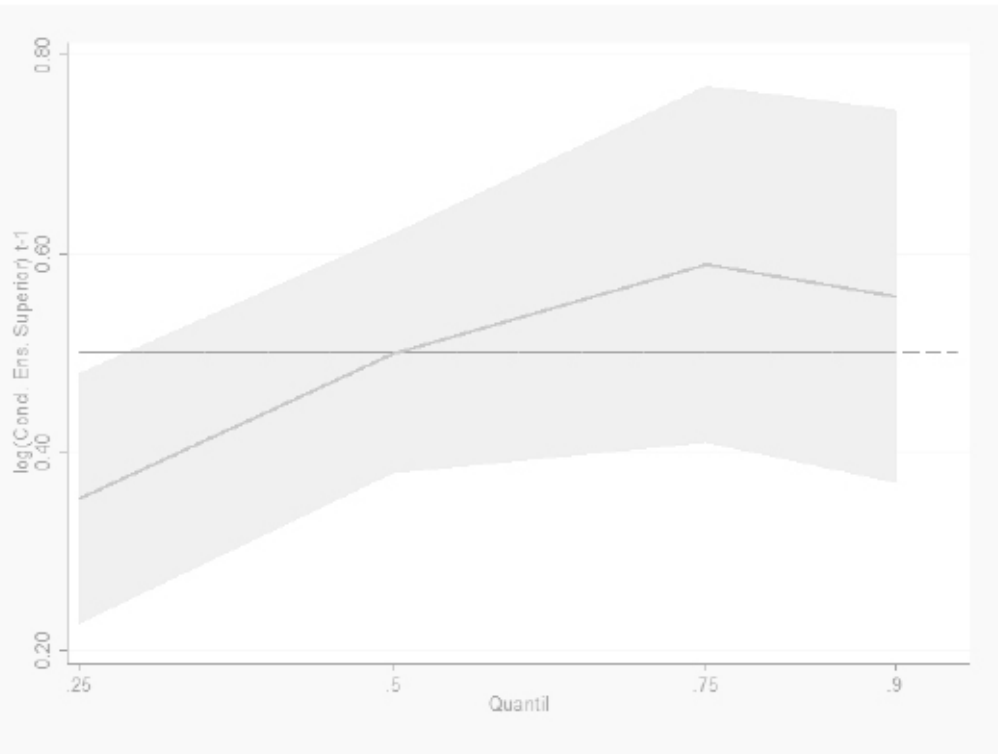

Fonte: Elaboração própria.

Este padrão de comportamento entre as elasticidades revela o impacto diferenciado dos recursos no crescimento dos estados. Conforme a dinâmica de cada economia, configurada pelos diferentes níveis de renda, os investimentos em infraestrutura podem apresentar um padrão de declínio, ao contrário de outros investimentos, como a formação do ensino superior e dos investimentos em CËT.

\subsection{Discussão com as Recentes Pesquisas}

O impacto diferenciado dos investimentos tem sido abordado recentemente em importantes estudos. Segundo Aghion, García-Peñalosa e Howitt (2004), muitas economias em desenvolvimento precisam tirar proveito do progresso tecnológico gerado em outros lugares. Para isto, uma determinanda economia precisar investir em educação e na provisão de bens públicos, como infraestrutura. $\mathrm{O}$ investimento equilibrado, especialmente na educação, pode conduzir as economias de baixa renda a uma taxa de crescimento maior em relação às economias de alta renda. ${ }^{10}$

10 "The comparison between Latin America and Southeast Asia also sheds interesting light on the relationship between growth and the organization of education in lower-income countries. In particular, the excessive emphasis on higher education and basic research at the expense of primary/secondary education in Latin 
No contexto da política de educação, recentementeVandenbussche, Aghion e Meghir (2006) demonstraram que os esforços públicos em investimentos na composição da educação dependem do estágio de desenvolvimento da economia para garantir o melhor uso do recurso da geração de um rápido crescimento. Nas economias mais afastadas da fronteira, com baixa atividade econômica e renda, o custo de oportunidade do fator de produção com alta qualificação (mão de obra com ensino superior) apresenta ser relativamente mais alto. Isso implica uma mobilidade do fator das regiões pobres para as mais desenvolvidas. Nesse sentido, tais investimentos podem não refletir em um crescimento imediato na renda, especialmente quando a demanda por este fator é menor nas regiões menos desenvolvidas (AGHION; HOWITT, 2009).

Nesta conjuntura, os investimentos em infraestrutura nas economias menos desenvolvidas podem criar oportunidades de negócios que repercutem em uma demanda gradual pelo fator qualificado. Isto porque a tecnologia vigente nestas economias podenão demandar fatores com alta qualificação. Em uma contribuição recente sobre este tema, Caselli e Coleman (2006) mostraram que a tecnologia apresenta um padrão complementar com o fator trabalho. Nas economias mais avançadas, a expansão da mão de obra com qualificação gera um rápido crescimento porque boa parte da tecnologia disponível se complementa de forma mais adequada a este tipo de fator. Desta forma, os investimentos em Pesquisa $\mathcal{E}$ Desenvolvimento podem apresentar um padrão heterogêneo no crescimento, na medida em que distinguimos as economias próximas ou mais afastadas da fronteira tecnológica.

Outras importantes pesquisas vêm sinalizando para este comportamento, destacando-se Acemoglu (2009), Wu (2010), Ang, Madsen e Islam (2011), Ott e Soretz (2011) e Coad (2011). Em todos os estudos destacados, as diferenças observadas entre as economias condicionam os resultados da política, de forma que os aspectos globais da tecnologia e do desenvolvimento são importantes fatores que precisam estar internalizados no desenho da política.

Outro importante estudo consiste na contribuição de Kateja (2012). Conforme a autora, a partir da década de 1990 as economias emergentes, em especial os BRIC's, implementaram importantes políticas que repercutiram diretamente na demanda por infraestrutura, como a abertura dos mercados em determinadas economias. Este processo de mudança da política teve impactos importantes na implementação de projetos em infraestrutura não apenas pelo setor público, mas também na participação do setor privado que vem crescendoespecialmente nas economias emergentes. Este processo, na década seguinte, alterou significativa-

American countries such as Mexico or Brazil may partly explain why these countries have underperformed in comparison to the East Asian tigers, where education has remained somewhat less elitist." (AGHION; GARCÍA-PEÑALOSA; HOWITT, 2004, p. 63). 
mente a composição dos investimentos, alterando a participação do estado na alocação dos recursos para tais investimentos.

A atuação do Estado ofertando uma infraestrutura para inserção do capital privado também é analisada por Farhadi (2015). Conforme o autor, países desenvolvidos, como as economias mais industrializadas na OECD, alavancaram um importante crescimento em mais de um século por meio de importantes investimentos públicos na construção de uma infraestrutura propícia à absorção do capital. Estes investimentos foram responsáveis por avanços significativos tanto na produtividade da mão de obra quanto na produtividade total dos fatores das firmas inseridas. Segmentos privados que realizam investimentos em infraestrutura apresentam retornos positivos, contudo, não superiores às externalidades envolvidas pelo avanço da infraestrutura (FERNANDÉZ; MONTUENGA-GÓMEZ, 2003).

Nesta direção, outros estudos como Angelopoulos, Economides e Kammas (2007), Egert, Kozluk e Sutherland (2009) e Crafts (2009) convergem em conclusões similares. Crafts (2009) estima que os investimentos em infraestrutura apresentam um coeficiente de elasticidade médio próximo a 0,2 em relação ao PIB. Embora a elasticidade estimada tenha demonstrado ser baixa, este aspecto pode estar relacionado ao efeito que os impostos exercem reduzindo a renda disponível. Analisando o cenário britânico, os investimentos apresentam uma grande relação benefícios/custos, contudo com retornos decrescentes na medida em que a infraestrutura venha a apresentar padrões de desenvolvimento. Isto sugere baixos coeficientes de elasticidade nas regiões desenvolvidas e elevadas nas economias em desenvolvimento, retratando um efeito heterogêneo conforme diferentes realidades econômicas. As evidências empíricas e teóricas retratadas no estudo convergem para a presente pesquisa.

Embora este processo tenha se apresentado de forma bastante visível no Brasil, isto não gerou o resultado esperado com profundas melhorias na infraestrutura local. Conforme Montes e Reis (2011), a clara redução da participação do investimento público, em decorrência tanto das privatizações quanto do ajuste fiscal, ao longo das duas últimas décadas tem limitado o crescimento nos estados brasileiros. Isso porque boa parte dos projetos de investimentos depende de um conjunto de instrumentos públicos que reforçam a eficácia destes projetos. Gargalos referentes à má qualidade dos gastos públicos, das assimetrias regionais, da insegurança jurídica, das deficiências na educação, da alta carga tributária, da corrupção, dentre outros fatores, restringem importantes projetos de investimento do setor privado na infraestrutura local. Esta restrição, vinculada à redução do investimento público, ainda com importantes projetos como o PAC, tem criado um grande impasse na trajetória econômica do país.

Desta forma, o cenário vigente de retração, em decorrência dos ajustes fiscais e de uma precária qualidade dos gastos, vem reforçando a necessidade de uma 
mudança na estratégia da política econômica. Tais fatores, vinculados às grandes assimetriais regionais dentro do país, limitam os esforços, caso a tomada de decisão desconsidere a influência das disparidades entre os estados na eficácia dos investimentos e da própria política. Os resultados apresentados por esta pesquisa corroboram tais estudos, apresentandoespecialmente este padrão de comportamente dentro do Brasil, quando a realidade se configura de forma tão heterogênea entre os estados e as regiões.

\section{Considerações Finais}

O presente estudo analisou o impacto dos investimentos em infraestrutura, além de outras importantes políticas, na geração de crescimento dos estados brasileiros, incorporando diferentes contextos de desenvolvimento. Na proposta teórica apresentada, os investimentos públicos atuam como um importante insumo produtivo, seguindo as assunções teóricas em Barro (1990) e Alesina e Rodrik (1994). Este aumento da produção gera uma acúmulode capital no tempo, conduzindo a economia em direção ao estado estacionário.

Adaptando a hipótese de Ortiz-Moctezuma et al. (2010), o aumento do crescimento cria novas demandas por infraestrutura que reforçam o crescimento. Ao contrário dos autores, somente uma parte do crescimento implica novas demandas, de maneira que, nas economias de baixa renda, o rápido crescimento pode ser sustentado com importantes projetos de infraestrutura, atraindo novas firmas e reduzindo os custos de transação associados a uma logista precária. A solução do problema dinâmico mostra que o estoque de capital de longo prazo é afetado positivamente pelos investimentos em infraestrutura.

Para testar as hipóteses levantadas pelo modelo teórico, construiu-se um modelo econométrico com dados em painel, empregando o método de estimação por regressão quantílica. Os resultados revelaram que os investimentos em infraestrutura têm importância relativamente maior nos estados de baixa renda ou, pelo menos, contribuem para um crescimento notoriamente maior nestes estados.

Ao contrário dos gastos em infraestrutura, a elasticidade dos gastos em CËT apresenta uma clara reversão à média. Nos quantis condicionais inferiores, a elasticidade parcial deste investimento é inferior em relação à média obtida por efeitos fixos. Estes resultados apontam que, nos estados de alta renda, o aumento dos investimentos em CEST contribui para um crescimento superior na renda, em relação aos estados de baixa renda. Este padrão também é observado quando analisamos o número de concluintes no ensino superior. Comparando os quantis de $25 \%$ e $75 \%$, a elasticidade parcial do número de concluintes no ensino superior salta de $0,353 \%$ para $0,557 \%$, respectivamente, totalizando uma variação de $157,79 \%$. 
Corroborando com recentes pesquisas, as implicações apresentadas mostram que determinadas políticas podem conduzir resultados diferenciados conforme a dinâmica de cada economia. Dadas as restrições orçamentárias e as metas pró-crescimento, um importante planejamento do estado que envolve a aplicação de recursos destinados a obter um máximo crescimento, deve incorporar na análise os diferentes contextos de desenvolvimento. A ausência deste instrumento de análise na tomada de decisão pode conduzir a uma alocação ineficiente dos recursos.

\section{Referências}

ACEMOGLU, D. Introduction to modern economic growth. Princeton, New Jersey: Princeton University Press, 2009.

ACEMOGLU, D.; AGHION, P.; ZILIBOTTI, F. Distance to frontier, selection and economic growth. Journal of the European Economic Association, v. 4, n. 1, p. 37-74, 2006.

AGHION, A.; SCHANKERMAN, M. Competition, entry and the social returns to infrastructure in transition economies. Economics of Transition, v. 7, n. 1, p. 79-101, 1999.

AGHION, P.; HOWITT, P. The Economics of growth. Cambridge, Massachusetts: The MIT Press, 2009.

AGHION, P.; GARCÍA-PEÑALOSA, C.; HOWITT, P. Knowledge and development: a schumpeterian approach. In: DOWRICK, S.; PITCHFORD, R.; TURNOVSKY, S. (Ed.). Economic growth and macroeconomic dynamics. New York: Cambridge University Press, 2004. p. 46-79.

ALESINA, A.; RODRIK, D. Distributive politics and economic growth. The Quarterly Journal of Economics, v. 109, n. 2, p. 465-490, 1994.

ANDREWS, D.; BUCHINSKY, M. Evaluation of a three-step method for choosing the number of bootstrap repetitions. Journal of Econometrics, v. 103, n. 1-2, p. 345-386, 2001.

ANG, J.; MADSEN, J.; ISLAM, M. The effects of human capital composition on technological convergence. Journal of Macroeconomics, v. 33, n. 3, p. 465-476, 2011.

ANGELOPOULOS, K.; ECONOMIDES, G.; KAMMAS, P. Tax-spending policies and economic growth: theoretical predictions and evidence from the OECD. European Journal of Political Economy, v. 23, n. 4, p. 885-902, 2007.

BARRO, R. Government spending in a simple model of economic growth. Journal of Political Economy, v. 98, n. 5, p. S103-S125, 1990.

BARRO, R.; SALA-I-MARTIN, X. Economic growth. 2. ed. Cambridge, MA: The MIT Press, 2004.

BUCHINSKY, M. Changes in US Wage Structure 1963-87: an application of quantile regression. Econometrica, v. 62, n. 2, p. 405-458, 1994. 
BUTTON, K. Infrastructure investment, endogenous growth and economic convergence. The Annals of Regional Science, v. 34, n. 1, p. 145-162, 1998.

CAMERON, A.; TRIVEDI, P. Microeconometrics: methods and applications. New York: Cambridge University Press, 2005.

CAPUTO, M. Foundations of dynamic economic analysis. New York: Cambridge University Press, 2005.

CASELLI, F.; COLEMAN, W. The world technology frontier. American Economic Review, v. 96, n. 3, p. 499-522, 2006.

CHATTERJEE, S.; MORSHED, A. Infrastructure provision and macroeconomic performance. Journal of Economic Dynamics \& Control, v. 35, n. 8, p. 1288-1306, 2011.

$\mathrm{COAD}, \mathrm{A}$. Appropriate business strategy for leaders and laggards. Industrial and corporate change, v. 20, n. 4, p. 1049-1079, 2011.

COAD, A.; RAO, R. Innovation and market value: a quantile regression analysis. Economics Bulletin, v. 15, n. 13, p. 1-10, 2006.

The firm-level employment effects of innovations in high-tech US manufacturing industries. Journal of Evolutionary Economics, v. 21, n. 2, p. 255-283, 2011.

CRAFTS, N. Transport infrastructure investment: implications for growth and productivity. Oxford Review of Economic Policy, v. 25, n. 3, p. 327-343, 2009.

EASTERLY, W.; SERVEN, L. The limits of stabilization. Stanford: Stanford University Press, 2003.

EFRON, B. Bootstrapping methods: another look at the jackknife. Annals of statistics, v. 7, n. 1, p. 1-26, 1979.

EFRON, B.; TIBSHIRANI, R. An introduction to the bootstrap. New York: Chapman $\mathcal{E}$ Hall, 1993.

EGERT, B.; KOZLUK, T.; SUTHERLAND, D. Infrastructure investment: links to growth and the role of public policies. Paris: OECD Economics Department, 2009. (Working paper, n. 686).

ESTACHE, A. Emerging infrastructure policy issues in developing countries: a survey of the recent economic literature. Washington, DC: The World Bank, 2004. (Working paper, n. 3442).

FARHADI, M. Transport infrastructure and long-run economic growth in OECD countries. Transportation Research Part A, v. 74, p. 73-90, 2015.

FEDDERKE, J.; PERKINS, P.; LUIZ, J. Infrastructural investment in long-run economic growth: South Africa 1875-2001. World Development, v. 34, n. 6, p. 1037-1059, 2006.

FERNANDÉZ, M.; MONTUENGA-GÓMEZ, V. The effects of public capital on the growth in Spanish productivity. Contemporary Economic Policy, v. 21, n. 3, p. 383-393, 2003. 
GREENE, W. Econometric Analysis. 7. ed. Boston, MA: Prentice Hall, 2012.

GRIFFITH, R.; REDDING, S.; VAN REENEN, J. Mapping the two faces of RED: productivity growth in a panel of OECD industries. Review of Economics and Statistics, v. 86, n. 4, p. 88395, 2004.

KATEJA, A. Building infrastructure: private participation in emerging economies. Social and Behavioral Sciences, v. 37, p. 368-378, 2012.

KOENKER, R. Quantile regression. New York: Cambridge University Press, 2005.

KOENKER, R.; BASSETT, G. Regression quantiles. Econometrica, v. 46, n. 1, p. 33-50, 1978.

KOENKER, R.; HALLOCK, K. Quantile regression. Journal of Economic Perspectives, v. 15, n. 4, p. 143-156, 2001.

LIN, J.; DOEMELAND, D. Beyond keynesianism: global infrastructure investments in times of crisis. Washington, DC: The World Bank, 2012. (Working paper, 5940).

LUCAS, R. Making a miracle. Econometrica, v. 61, n. 2, p. 251-272, 1993.

MONTES, G.; REIS, A. Investimento público em infraestrutura no perído pós-privatizações. Revista Economia 8 Sociedade, v. 20, n. 1, p. 167-194, 2011.

ORTIZ-MOCTEZUMA, M. et al. Development of transportation infrastructure in the context of economic growth. In: CUARESMA, J.; PALOKANGAS, T.; TARASYEV, A. (Ed.). Dynamic systems, economic growth, and the environment. New York: Springer, 2010.

OTT, I.; SORETZ, S. Public policies and convergence. Journal of Economic Dynamics 8 Control, v. 35, p. 1435-1450, 2011.

QUAH, D. Twin peaks: growth and convergence in models of distribution dynamics. The Economic Journal, v. 106, n. 437, p. 1045-1055, 1996.

ROCHA, L.; SILVEIRA, J. Fronteira tecnológica, políticas públicas e o crescimento dos estados brasileiros. In: ENCONTRO NACIONAL DE ECONOMIA, 37., 2009, Foz do Iguaçu. Anais... Foz do Iguaçu: ANPEC, 2009.

ROCHA, L.; KHAN, A.; LIMA, P. Construindo competências tecnológicas na agricultura brasileira: articulando a política nacional de inovação com os investimentos em infraestrutura. In: ENCONTRO REGIONAL DE ECONOMIA/FÓRUM BNB DE DESENVOLVIMENTO, 18., 2013, Fortaleza. Anais... Fortaleza: ANPEC/BNB, 2013.

VANDENBUSSCHE, J.; AGHION, P.; MEGHIR, C. Growth, distance to frontier and composition of human capital. Journal of Economic Growth, v. 11, n. 2, p. 97-127, 2006.

WU, H. Distance to frontier, intellectual property rights, and economic growth. Economics of Innovation and New Technology, v. 19, n. 2, p. 165-183, 2010. 


\section{Apêndice A - Apêndice Matemático}

O problema de otimização é dado pela solução em:

$$
\begin{aligned}
& \underbrace{\operatorname{máx}}_{c \geq 0} J[c, k, z, t] \stackrel{\text { def }}{=} \int_{0}^{+\infty} e^{-\rho t} \cdot u(c) \cdot d t \\
& \text { s.a. }\left\{\begin{array}{cc}
\dot{k}=\varphi(k, G, c)=f(k, G)-c-d k & \\
\dot{z}=\vartheta(g, z)=\mu g-\delta z & \\
k(0)=k_{o} & \\
z(0) & =z_{o} \\
c(0) & =c_{o} \\
d>\delta & \\
t \in[0,+\infty) & \\
\forall(c, k, z) \in \operatorname{int}\{\mho \mho \times \mathcal{K} \times Z\} &
\end{array}\right. \\
& c:[0,+\infty) \rightarrow \mho ; k:[0,+\infty) \rightarrow \mathcal{K} ; z:[0,+\infty) \rightarrow Z \\
& \lambda_{k}:[0,+\infty) \rightarrow \mathbb{R}_{+} ; \lambda_{z}:[0,+\infty) \rightarrow \mathbb{R}_{+}
\end{aligned}
$$

Para uma solução factível, o problema AM.1 deve satisfazer o teorema:

TEOREMA1: Considerando o Problema de Otimização AM.1, sujeito às restrições de forma, em que $\varphi($. ) e $\vartheta($. ) sejam funções continuamente diferenciáveis em relação aos seus argumentos e supondo que este problema tem uma solução contínua de interior $(\hat{c}, \hat{k}, \hat{z}) \in \operatorname{int}\{\gamma \times \mathcal{K} \times z\}$, dada a trajetória da variável estado $k(t)$, então existe uma função diferenciável (co-estado) $\lambda_{k}(t) e \lambda_{z}(t)$ e definida em $t \in[0,+\infty)$.

A partir do teorema acima, podemos definir uma função hamiltoniana de valor corrente que satisfaz esta condição necessária. Esta função é continuamente diferenciável em relação aos seus argumentos.

$$
\mathcal{H}\left[c, k, z, \lambda_{k}, \lambda_{z}\right]=u(c)+\lambda_{k}[\varphi(k, G, c)]+\lambda_{z}[\vartheta(g, z)]
$$

O princípio do máximo consiste na solução que maximiza a função hamiltoniana e é definido a partir do teorema a seguir:

TEOREMA 2 (Principio do Máximo): Dada a solução de interior $(\hat{c}, \hat{k}, \hat{z}) \in \operatorname{int}\{\mho \times \mathcal{K} \times \mathcal{Z}\}$, e a trajetória correspondente das variáveis estado $(\hat{k}, \hat{z}) \in \operatorname{int}\{\mathcal{K} \times Z\}$, então existe uma solução ao controle ótimo que deve satisfazer as condições necessárias:

$$
\begin{gathered}
\frac{\partial}{\partial c} \mathcal{H}\left[c, k, z, \lambda_{k}, \lambda_{z}\right] \equiv 0 \\
\dot{\lambda}_{k}=\rho \lambda_{k}-\frac{\partial}{\partial k} \mathcal{H}\left[c, k, z, \lambda_{k}, \lambda_{z}\right]
\end{gathered}
$$




$$
\begin{gathered}
\dot{\lambda}_{z}=\rho \lambda_{z}-\frac{\partial}{\partial z} \mathcal{H}\left[c, k, z, \lambda_{k}, \lambda_{z}\right] \\
\dot{k}=\frac{\partial}{\partial \lambda_{k}} \mathcal{H}\left[c, k, z, \lambda_{k}, \lambda_{z}\right] \\
\dot{z}=\frac{\partial}{\partial \lambda_{z}} \mathcal{H}\left[c, k, z, \lambda_{k}, \lambda_{z}\right]
\end{gathered}
$$

Condições de Transversalidade:

$$
\begin{aligned}
& \lim _{t \rightarrow+\infty} e^{-\rho t} \lambda_{k} k=0 \\
& \lim _{t \rightarrow+\infty} e^{-\rho t} \lambda_{z} z=0
\end{aligned}
$$

Havendo pontos factíveis ao presente problema, a unicidade da solução é garantida quando a função hamiltoniana é estritamente côncava, de forma que satisfaz a desigualdade a seguir:

$$
\mathcal{H}\left[\hat{c}, \hat{k}, \hat{z}, \lambda_{k}, \lambda_{z}\right] \geq \mathcal{H}\left[c, k, z, \lambda_{k}, \lambda_{z}\right] \therefore \forall(\hat{c}, \hat{k}, \hat{z}) \in \operatorname{int}\{\mho \widetilde{K} \times \mathcal{K} \times z\} \text { AM. } 3
$$

A solução para $\hat{k}$ que satisfaz AM.3 é dada por:

$$
\hat{k}=\left[\frac{\alpha}{d-\delta}\right]^{\frac{1}{1-\alpha}} G
$$

Seguindo a solução para $\hat{C}$ :

$$
\begin{gathered}
f(\hat{k}, G)-\hat{c}-d \hat{k}=0 \\
\hat{c}=\Omega(\alpha, d, \delta) G \geq 0 \\
\Omega(\alpha, d, \delta) \equiv\left[\left[\frac{\alpha}{d-\delta}\right]^{\frac{\alpha}{1-\alpha}}-d\left[\frac{\alpha}{d-\delta}\right]^{\frac{1}{1-\alpha}}\right]>0 \Leftrightarrow d(1-\alpha)>\delta
\end{gathered}
$$

Como a elasticidade parcial dos gastos em infraestrutura satisfaz a Lei dos Rendimentos Decrescentes, logo:

$$
\begin{array}{cc}
(1-\alpha) \in(0,1) \therefore \forall \alpha \in(0,1) \rightarrow(1-\alpha)<1 & \text { (Restrições } \\
\frac{\delta}{d}<1 \Leftrightarrow d>\delta & \text { do Modelo) }
\end{array}
$$

Em cada solução, os gastos em infraestrutura afetam positivamente, de maneira que a política de infraestrutura afeta a longo prazo tanto o estoque de capital quanto os padrões de consumo. Assim, coeteris paribus, as diferenças entre as variáveis no longo prazo podem ser explicadas pelos diferentes instrumentos da política. 
Tomando duas economias diferentes, temos:

$$
\frac{\hat{k}_{j}}{\hat{k}_{j^{\prime}}}=\frac{\Psi(\alpha, d, \delta) G_{j}}{\Psi\left(\alpha^{\prime}, d^{\prime}, \delta^{\prime}\right) G_{j \prime}} ; j \neq j^{\prime}
$$

A partir da equação AM.4, as diferenças nos investimentos em infraestrutura repercutem em níveis diferenciados de estoque de capital. Economias que direcionaram maiores níveis de investimentos administram maiores níveis de estoque de capital, elevando consequentemente a taxa de crescimento em direção ao estado estacionário.

Este padrão também é observado quando comparamos os diferentes níveis de consumo a longo prazo:

$$
\frac{\hat{c}_{j}}{\hat{c}_{j \prime}}=\frac{\Omega(\alpha, d, \delta) G_{j}}{\Omega(\alpha \prime, d \prime, \delta \prime) G_{j \prime}} ; j \neq j^{\prime}
$$

AM.5

Controlando os parâmetros do modelo, níveis maiores de investimento em infraestrutura repercutem em maior níveis de consumo, aumentando a taxa de crescimento em direção ao estado estacionário. Este exercício também pode ser ampliado incluindo o efeito da tecnologia nas diferenças entre as variáveis.

Recebido em: 07/04/2015. Aceito em: 06/09/2016. 